\title{
Three-dimensional planning-guided total hip arthroplasty through a minimally invasive direct anterior approach. Clinical outcomes at five years' follow-up
}

\author{
Elhadi Sariali $^{1,2}$ (D) Yves Catonne $^{1} \cdot$ Hugues Pascal-Moussellard $^{1}$
}

Received: 26 February 2016 / Accepted: 5 June 2016 / Published online: 16 June 2016

(C) The Author(s) 2016. This article is published with open access at Springerlink.com

\begin{abstract}
Purpose The direct anterior approach is an attractive option for total hip arthroplasty (THA) in order to achieve a quicker rehabilitation. However, this surgical technique presents a longer learning curve and a higher complications rate compared with the standard approach. We investigated whether three-dimensional (3D) planning anticipated the surgical difficulties and helped to achieve a low complications rate with respect to intra-operative complications, dislocation risk and lower limb discrepancy (LLD).

Methods One hundred and fifty-four consecutive patients underwent a primary cementless THA using a direct anterior approach. A 3D planning was performed in order to anticipate the difficulties that may be encountered regarding femur perforation or fracture, dislocation and LLD. All patients were assessed at a mean five years' follow-up.

Results No false route and no fracture occurred at the time of surgery. All the surgical difficulties were anticipated. A motorised reaming procedure of the femur was required in six patients because of a very dense bone or a narrow femur. A retroverted neck was used in $7 \%$ of patients because of a torsional abnormality and enabled an increase in stability. The real implant sizes were the same as the ones planned in $97 \%$ for the cup, $96 \%$ for the stem and $100 \%$ for the neck. At five years' follow-up, no dislocation occurred, no patient
\end{abstract}

Electronic supplementary material The online version of this article (doi:10.1007/s00264-016-3242-z) contains supplementary material, which is available to authorised users.

Elhadi Sariali

hedisari@yahoo.fr

Hôpital Pitié Salpêtrière, 47-83 Bd de l'Hôpital, 75013 Paris, France

2 Laboratoire d'imagerie Biomédicale, 15 rue des cordeliers, 75005 Paris, France complained about LLD and excellent clinical outcomes were achieved.

Conclusions This study demonstrates that $3 \mathrm{D}$ pre-operative planning-guided THA through a minimally invasive direct anterior approach is a safe and accurate procedure.

Level of Evidence: Level IV

Keywords Three-dimensional $\cdot$ Planning $\cdot$ Direct anterior approach $\cdot$ Dislocation $\cdot$ Accuracy

\section{Introduction}

The direct anterior approach (DAA) is an attractive option for total hip arthroplasty (THA) in order to decrease the dislocation rate [1] and to achieve a quicker rehabilitation [2]. However, this surgical technique presents a longer learning curve and a higher complications rate, especially on the femoral side including femoral stem malposition [3] and intraoperative femur fractures [4-7].

Many authors reported that accurate pre-operative planning is necessary to avoid complications $[8,9]$. However, the conventional two-dimensional (2D) templating has a relatively low accuracy despite a correct image calibration $[10,11]$. With respect to the components size and position, an excellent accuracy was reported with a novel three-dimensional (3D) pre-operative planning technique as the difficulties likely to be encountered were detected and solved pre-operatively by adapting the surgical procedure and choosing the optimal implants which would best restore the hip biomechanics [11-14]. However, it is not clear whether this technique helped to decrease the complications rate with respect to intra-operative fractures, dislocation risk and complaints about lower limb discrepancy (LLD). Furthermore, no clinical results have yet been reported for this technique. 
Our hypothesis was that 3D pre-operative planning anticipates the technical difficulties likely to be encountered during the surgical procedure, consequently allowing a safe and accurate surgical procedure to be achieved when using a DAA. We therefore asked whether the 3D planning (1) was accurate, (2) allowed the anticipation of surgical difficulties and (3) helped to achieve a low complications rate with respect to intra-operative complications, LLD and dislocation risk at five years of follow-up.

\section{Materials and methods}

A prospective non-comparative non-randomised study was carried out between January 2009 and November 2011. One surgeon performed 154 consecutive 3D planning-assisted total hip replacements using an anatomical, proximally hydroxyapatite (HA)-coated, cementless, modular-neck stem (SPS-Modular®; Symbios, Yverdon-les-Bains, Switzerland) and an HA-coated acetabular component (APRIL $®$; Symbios) with a delta alumina ceramic head and liner (Ceramtec, Plochingen, Germany). The head diameter was $32 \mathrm{~mm}$ for cup diameters under $50 \mathrm{~mm}$ and $36 \mathrm{~mm}$ otherwise.

All the surgical procedures were performed by one surgeon, who used a minimal invasive direct anterior approach on a fracture table. The surgeon was trained during his fellowship to the standard DAA. This study included his learning curve as an independent senior surgeon. The patients were composed of 57 women and 97 men, with a mean age at the time of surgery of $58.8 \pm 13.5$ years (18-84) and a mean body mass index (BMI) of $26.5 \pm 4.4 \mathrm{~kg} / \mathrm{m}^{2}(17-35.5), 63$ patients (41\%) were then over weighted and $28(15 \%)$ were obese. The aetiologies are shown in Table 1.

Prior to surgery, patients were subject to $3 \mathrm{D}$ planning in order to determine the prosthetic component's size and position, as well as to anticipate the surgical difficulties [13]. Planning and surgery simulation was performed using the HIP-PLAN® software, which is based on low-dose computed tomography (CT) scans [15]. Matching of pre-operative and post-operative CT scans was performed by aligning the bone landmarks in order to compare the post-operative hip anatomy to the planned one.

\section{Pre-operative planning}

In regards to the cup, the goal was to restore the native acetabular anteversion and to achieve an inclination of $40^{\circ}$.

In order to analyse the alteration of the hip anatomy induced by the arthroplasty, four points were determined (Fig. 1): (1) the centres of the native acetabulum (Ac) and the final cup $(\mathrm{Cc})$; the vector between these two points, $\overrightarrow{A c C c}$, was labelled the acetabular displacement; (2) the
Table 1 Aetiologies of the patients

\begin{tabular}{ll}
\hline Aetiology & Number of patients (\%) \\
\hline Primary osteoarthritis & $106(69 \%)$ \\
Avascular necrosis & $24(16 \%)$ \\
DDH & $6(4 \%)$ \\
Inflammatory arthritis & $5(3 \%)$ \\
Post-traumatic osteoarthritis & $4(3 \%)$ \\
Legg-Calve-Perthes disease & $5(3 \%)$ \\
Slipped capital femoral epiphysis & $2(1 \%)$ \\
Fused hip & $2(1 \%)$ \\
\hline
\end{tabular}

centres of the femoral head centre (FHc) and the femoral ball (FBc); the vector between these two points, $\overrightarrow{F H c F B C}$, was labelled the femoral head displacement. The global femoral displacement $\overrightarrow{F D}$ was measured as the sum of these two vectors, $\overrightarrow{A c C c}+\overrightarrow{F H c F B C}$. The goal was to achieve $\overrightarrow{F D}=\overrightarrow{0}$, which means that the relative positions of the two native centres, Ac and FHc, were not altered by the arthroplasty.

The objective was to restore the global offset corresponding to the sum of the acetabular offset and the femoral offset. If a medial translation of the cup was required in order to achieve a good cup stability and coverage, the femoral offset was increased by the same amount in order to maintain the same global offset (Fig. 2). Based on previously reported results regarding the dislocation risk of anterior THA, the goal was to restore the native femoral anteversion unless the femoral displacement (FD) in the antero-posterior direction was above $8 \mathrm{~mm}$. This situation was observed when were combined a posterior shift of the hip rotation centre (COR) and an increase in the femoral anteversion (the stem anteversion higher than the native femoral anteversion). In this case, a retroverted neck was used to make the femoral ball centre coincides with the cup centre (Fig. 3). The threshold of $8 \mathrm{~mm}$ was determined based on a previous biomechanical study [16] showing that dislocation after anterior THA was correlated to two factors: (1) a posterior translation of the hip rotation centre of 6-8 mm; (2) an increase in the femoral anteversion. The neck modularity allowed three different neck-shaft angles ( $8^{\circ}$ varus, straight, $8^{\circ}$ valgus), three anteversion angles $\left(15^{\circ}\right.$ retroversion, $0^{\circ}, 15^{\circ}$ anteversion) and two different lengths for each neck (short, long). The straight neck corresponded to a $134^{\circ}$ neck-shaft angle. For the femoral head, three lengths could be used: $-4 \mathrm{~mm}, 0$ and $+4 \mathrm{~mm}$.

At the time of surgery, in order to test the hip stability, the nurse was asked to rotate externally the hip in neutral hip extension using the traction table device. A hip was considered as perfectly stable if dislocation did not occur 


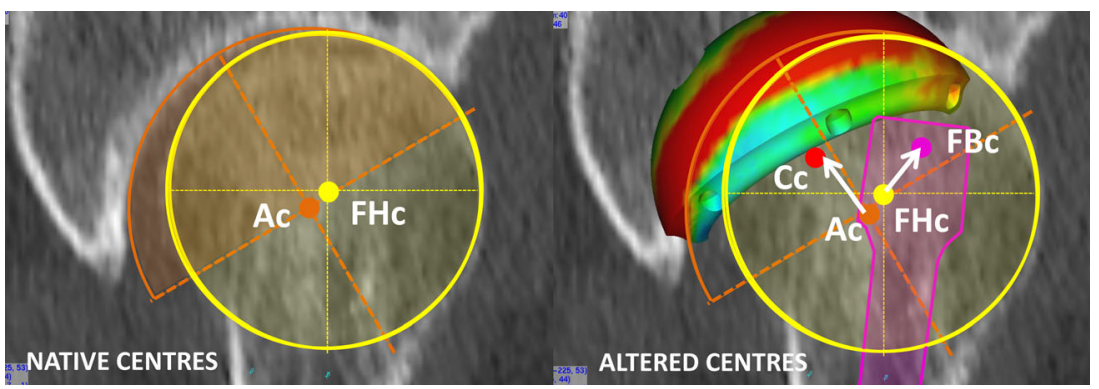

Fig. 1 The centres of the native acetabular $(A c)$ and femoral head $(F H c)$ were determined. The distance between these two points was labelled Initial displacement which corresponds to the wear. The centres of the final cup $(C c)$ and femoral ball $(F B c)$ were determined. The vector $\overrightarrow{A c C c}$ was labelled acetabular displacement. The vector $\overrightarrow{F H c F B C}$ was labelled

whatever was the external rotation. For the posterior instability, the foot was removed from the traction table and the test was performed by the surgeon in flexionadduction and internal rotation.

\section{Analysis of the accuracy of the hip reconstruction}

In order to determine the accuracy of the anatomy restoration, the following parameters were studied: (1) the coordinates of the hip rotation centre and the femoral head centre; (2) the 3D value of the lower limb lengthening; (3) the 3D value of the femoral off-set; (4) the acetabular anteversion and abduction angles; (5) The femoral anteversion angle.

\section{Anticipation of the surgical difficulties}

The surgeon tried to forecast the following difficulties. (1) A femoral perforation or a femur fracture which, in our experience, is likely to occur if three conditions are combined: (a) a

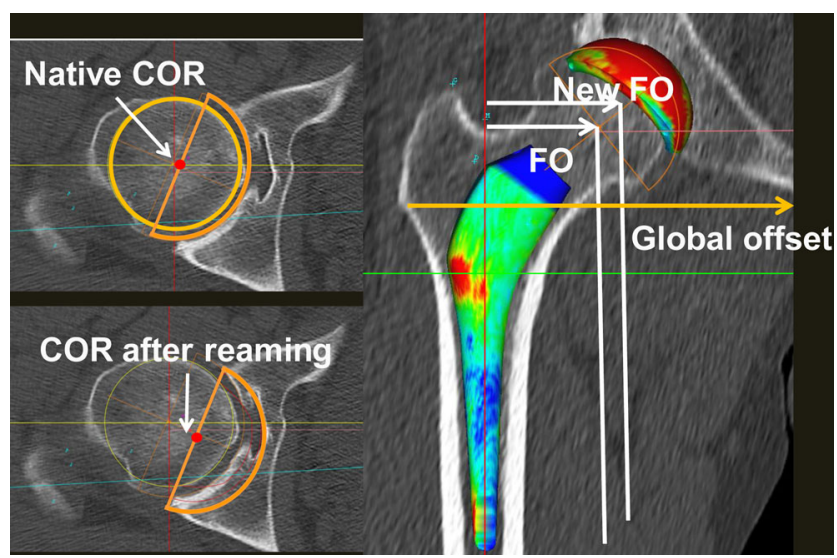

Fig. 2 In some cases, a medial displacement of the COR was required. In this case, the alteration of the COR was compensated by an increase in the femoral offset $(F O)$ in order to keep the global offset constant and to avoid hip dislocation femoral head displacement. The global femoral displacement $\overrightarrow{F D}$ was measured as the sum of these two vectors $\overrightarrow{A c C c}+\overrightarrow{F H c F B C}$. We aimed for $\overrightarrow{F D}=\overrightarrow{0}$

high anterior curvature of the femur, (b) a high density of the cancellous bone at the upper part of the femur and (c) a narrow femoral isthmus (Fig. 4). In these cases, before starting the rasping procedure, a femoral guide was implanted along the femur and a motorised reaming procedure was performed with flexible reamers. (2) The difficulties of restoring simultaneously the offset and the length, especially for patients who have a disproportion between the femoral canal size and the femoral offset (large femur and low offset and vice-versa). (3) The femur torsion abnormalities for which the final anteversion may be excessively increased (above $10^{\circ}$ ) or at contrary decreased $\left(10^{\circ}\right)$ comparatively to the native femoral anteversion (Fig. 3). For these cases, a retroverted or anteverted neck was proposed to increase the hip stability and to avoid dislocation.
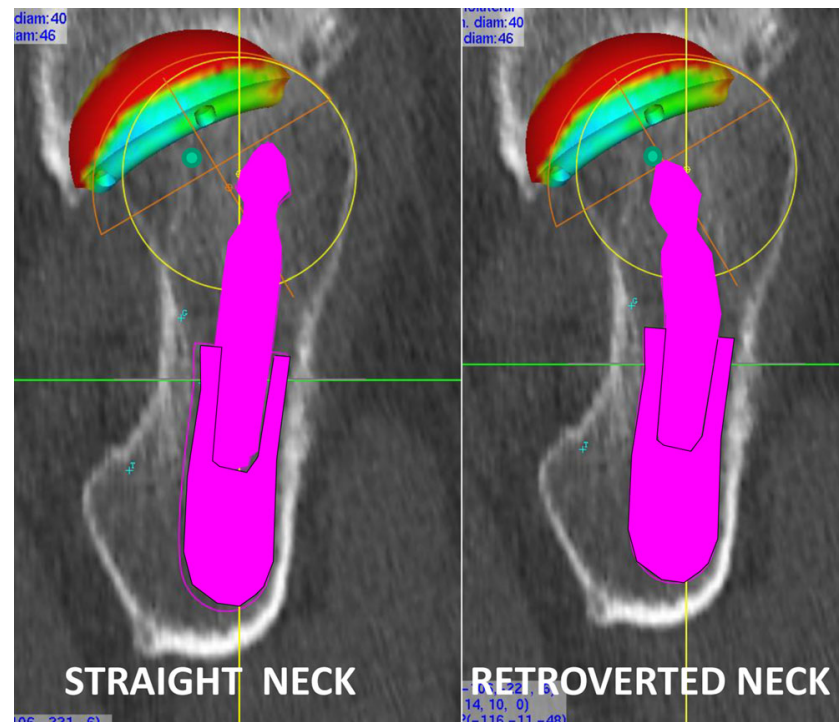

Fig. 3 A retroverted neck was used in $7 \%$ of patients in order to compensate for a posterior translation of the hip rotation centre induced by an excessive posterior reaming procedure required to achieve a good primary stability of the cementless cup. In this case the femoral anteversion was decrease in order to make the centres of the femoral ball and the cups spontaneously coincide 


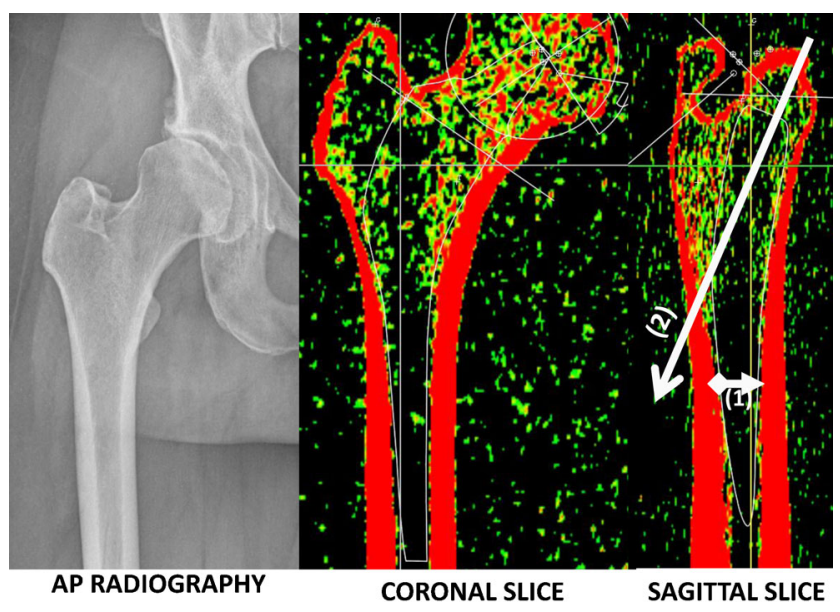

Fig. 4 The sagittal analysis of the femur showed some patients at risk of femoral perforation (arrow 2). These patients had a high proximal anterior curvature, a narrow isthmus (arrow 1) and an elevated bone density

\section{Clinical assessment}

All the patients were assessed clinically at a mean follow-up of $5 \pm 0.8$ years (ranging from 3.7 to 6.9 years) with the Harris hip score (HHS) and the Oxford quality of life score (OS). There were no losses to follow-up. At the last follow-up, two complications were analysed: dislocation and clinical complain about LLD.

The study was conducted according to the French bioethics law (Article L. 1121-1 of law no. 2004-806, 9 August 2004), and an approbation was accorded by the patient protection committee responsible for this hospital. Informed consent was obtained from all individual participants included in the study.

\section{Statistical analysis method}

Pearson's correlation coefficient was used to study the correlation between two variables. The accuracy of all the parameters was assessed as the mean difference between the planned values and the postoperative values (mean \pm standard deviation). The distribution of variables was tested for normality using the Ryan-Joiner and Shapiro-Wilk tests. For normally distributed variables, when two groups had the same variances, differences between them were analysed using Student's $t$-test. For abnormally distributed variables or normally distributed variables with different variances, we used the Mann and Whitney test. Regarding the LLD, the rate of outliers was analysed: less than $5 \mathrm{~mm}, 5-10 \mathrm{~mm}$ and more than $10 \mathrm{~mm}$ of LLD. A $p$ value of less than 0.05 was considered significant. Statistical analysis was performed with JMP software (version 11; SAS institute).

\section{Results}

\section{Accuracy analysis}

The implanted components were the same as the ones planned in $96 \%$ of cases for the stems, $100 \%$ for the necks, $97 \%$ for the cups and $95 \%$ for the heads.

We found no significant difference between the mean planned femoral anteversion value $\left(25.1^{\circ} \pm 9.8\right)$ and the post-operative one $\left(27.2^{\circ} \pm 9.9\right)(p=0.22)$, and a very high correlation between these two values was observed $(0.89$, $p=0.00001)$.

There was no significant difference between the mean planned cup anteversion $\left(17.5^{\circ} \pm 6.8\right)$ and the mean postoperative one $\left(19.7^{\circ} \pm 10\right)$. There was a significant correlation between the planned acetabular anteversion and the post-operative one (correlation coefficient 0.14 ). However, this correlation was lower for the cup than for the stem. There was no significant difference between the mean planned cup abduction $\left(41.1^{\circ} \pm 2.2\right)$ and the mean post $\left(40.8^{\circ} \pm 7\right)$.

The hip rotation centre was restored with an accuracy of $-0.5 \mathrm{~mm} \pm 3$ horizontally $-0.8 \mathrm{~mm} \pm 3$ laterally and $0.0 \mathrm{~mm} \pm 2.7$ sagittally. Limb length was restored with an accuracy of $-1.5 \mathrm{~mm} \pm 3.6$ and the femoral off-set with an accuracy of $0.8 \mathrm{~mm} \pm 2.6$. The LLD was less than $5 \mathrm{~mm}$ in $95 \%$ of cases, between 5 and $10 \mathrm{~mm}$ in $5 \%$ and above $10 \mathrm{~mm}$ in no patient.

\section{Safety analysis: anticipation of intra-operative difficulties}

Almost all the technical surgical difficulties were anticipated, especially with respect to the risk of femoral perforation, the peri-operative hip instability and the simultaneous restoration of the offset and the limb length. A motorised femoral reaming was scheduled and performed in six cases because of a very dense bone and a high anterior curvature of the femur that increased the risk of femoral perforation or fracture (Fig. 4). An $8^{\circ}$ varus femoral neck was used in $60 \%$ of patients either because of a varus morphotype or because of a decrease in the acetabular offset generated by the acetabular reaming procedure (Fig. 2).

All the cases of hip instability at the time of surgery were anticipated and solved by using retroverted necks as planned before surgery. Indeed, a retroverted neck was used in $7 \%$ of cases either because of an increased femoral anteversion (stem anteversion higher than the native anteversion) or a posterior translation of the hip rotation centre (Fig. 3). At the time of surgery, a straight neck was tested showing a hip instability that was solved (as planned) with the retroverted neck in all the cases. Finally, no femoral perforation and no peri-operative fractures occurred in this cohort. 


\section{Clinical assessment at the last follow-up}

No dislocation occurred in this cohort, and no patient complained about LLD at the last follow-up. The mean HHS at the last follow-up was $97 \pm 7$ (ranging from 59 to 100). The mean OS at the last follow-up was $57 \pm 6$ (ranging from 37 to $60)$. Two periprosthetic fractures occurred at 1 month and 2.5 years of follow-up. Both were females with severe osteoporosis (one sequella of cerebral stroke, one sequella of pelvic ring fracture with no weight bearing for 1 year). Both patients underwent reduction and internal osteosynthesis with stem retention. These two patients had very good results at the last follow-up. No patient was revised for loosening or deep infection. At an average five years' follow-up, no neck fractures occurred.

\section{Discussion}

The main finding of this study was that 3D planning-assisted THA is an accurate and safe surgical procedure when using a minimally invasive DAA. Excellent clinical outcomes were achieved, comparing well with the best reported studies for minimally invasive DAA THA. No mechanical complications occurred at the time of surgery, the patients experienced no dislocation at a mean five years of follow-up and none complained about clinical LLD. However, delayed proximal femur fracture occurred in two female patients, highlighting the limitation of a short metaphyseal cementless fixation in osteoporotic patients. These two patients were treated with an internal fixation and stem retention with good clinical outcomes. Given this risk, the authors now prefer to use cemented straight stems in female patients with severe osteoporosis. In order to anticipate the fracture risk at the time of the 3D planning, we have developed a numerical algorithm integrating the stem contact area and the bone density. A study is now being performed in order to validate our calculation method.

Lee and Marconi [4] shown that the most common complication following DAA THA were nerve dysfunction $(2.8 \%)$, intraoperative fractures $(2.3 \%)$ and dislocation $(1.2 \%)$. Homma et al. [7] reported $32 \%$ of lateral femoral cutaneous nerve injury with DAA that correlated to lower FJS-12 scores. Spaans et al. [6] reported also a high complication rate in the early experience of DAA THA, with no benefit shown when compared with the posterior approach. High complication rates were also reported by Jewett et al. [5] who shown intra-operative complications in 26 out of 800 patients including trochanteric fractures $(2.3 \%)$ and femoral perforation $(0.37 \%)$. These two complications are related in our experience to either a coxa vara morphotype or a high anterior curvature of the proximal femur that requires a more important posterolateral release that may be difficult to achieve, especially in the early experience.

We observed no intra-operative fractures and no perforations, despite the fact that this cohort included the surgeon's learning curve. We found two explanations for these good results. First, the use of the SPS stem makes easier the rasping procedure as well as the introduction of the femoral stem through the osteotomy plane because of its anatomic shape that follows the proximal sagittal curvature of the femur (Fig. 5). Second, the 3D planning detects risky patients who combine a high cancellous bone density, an elevated anterior femur curvature and a narrow femoral isthmus. Based on the $3 \mathrm{D}$ planning, the
Fig. 5 The use of an antomic stem (SPS) that is designed with an anterior curvature and torsion decreases the risk of femoral perforation (a) and trochanteric fracture (b) at the time of the rasping procedure comparative to straight stems a

STRAIGHT STEM

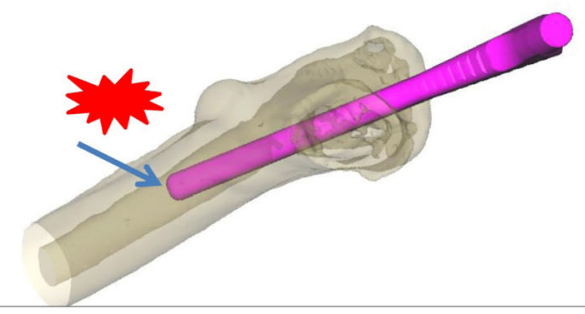

b

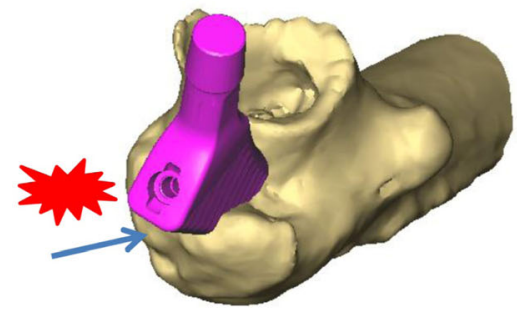

ANATOMIC STEM

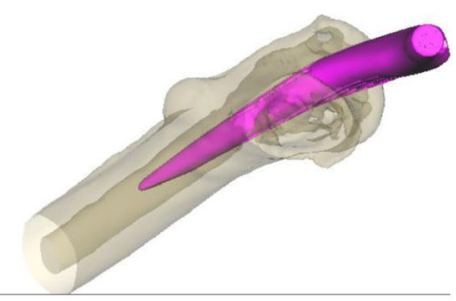

ANATOMIC STEM

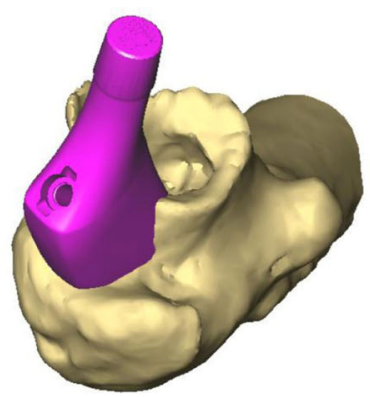


authors anticipated this intra-operative difficulty and sorted it out with using the SPS stem and a motorised reaming procedure.

The 3D planning-guided THA showed a high accuracy, comparing well with the previously reported studies about the 3D planning methodology using the Hip-Plan software [11-13]. Furthermore, contrary to navigation or fluoroscopic guidance techniques [17], 3D planning uses only local anatomic landmarks and can be used as easily for all surgical approaches and regardless of the patient's position. Another strong point of this study is the fact that the cohort was consecutive, prospectively analysed and with no lost patient at an average five years of follow-up.

On the other hand, the most obvious limitation of this study was that all patients were operated on by the same surgeon. However, this cohort included his learning curve, suggesting that 3D planning may help decrease the intra-operative complication rate in the early experience of DAA. Indeed, with respect to the intra-operative complications, the learning curve varies according to the authors from 20 [18] to $200[1,5]$. In our future work, we will replicate this study with a variety of surgeons with different skill levels.

The 3D planning anticipated many complications. Firstly, the sagittal analysis of the femur showed some patients at risk of femoral perforation. The use of a flexible intra-femoral guide and a motorised reamer help to avoid perforation. Secondly, some patients with acetabular dysplasia required a postero-medial acetabular reaming that generated consequently a posterior translation of the hip rotation centre. This alteration induced an anterior instability of the hip that was sorted out with a retroverted neck. However, above $15^{\circ}$ of torsion abnormality, neck modularity does not allow a sufficient correction and other surgical strategies should be considered such as custom stems [19].

No dislocation occurred in this cohort and all the cases of peri-operative hip instability were detected before surgery, confirmed during surgery and sorted out with retroverted necks. We used only large head diameters ( 32 and $36 \mathrm{~mm}$ ) that decrease dislocation risk even though the $3 \mathrm{D}$ anatomy is not accurately restored. However, in this case, microseparation and edge loading may occur, generating consequently squeaking and ceramic fracture [20].

Despite the fact that we had no modular neck fracture, we stopped implanting modular neck stems given the risk of cavernous corrosion and fracture [21]. Consequently, a modified SPS stem (SPS Evolution; Symbios, Yverdon-les-Bains, Switzerland) was designed with a $5^{\circ}$ more varus and a $5^{\circ}$ more retroverted neck in order to avoid the use of modular necks. The SPS Evolution neck orientation and length were based on a post-operative 3D analysis of 600 patients that underwent THA with the SPS-modular stem. The patients were classified by femoral stem size, and for each stem size the 3D position of the acetabular centre was determined relative to the final post-operative stem shaft position inside the femur. The SPS Evolution neck was designed in order to aim at the centre of the points cloud. A study is now being performed in order to test whether the head modularity may be enough to address all the patients and otherwise to determine the outlier patients requiring a specific stem.

Before surgery, based on the 3D femur morphotype analysis, we chose either the cementless SPS Evolution stem or a cemented quadrangular straight Muller-type stem for severe osteoporotic patients. In some dysplastic femurs, such as severe coxa-vara or $\mathrm{DDH}$, a short metaphyseally fixed custom stem is used in order to insure a correct anchorage, bone preservation and an accurate hip reconstruction [19].

\section{Conclusions}

Three-dimensional pre-operative planning-guided THA through a minimally invasive direct anterior approach is a safe and accurate surgical procedure. A very low complication rate was found, with no intra-operative fractures, no perforations, no dislocations and no complaints about LLD. The described procedure should improve the clinical outcome but we need long-term studies and higher numbers of patients to show this.

\section{Compliance with ethical standards}

Conflict of interests Intellectual property rights: royalties from Symbios SA

Funding information No funding sources were provided for this study

Open Access This article is distributed under the terms of the Creative Commons Attribution 4.0 International License (http:// creativecommons.org/licenses/by/4.0/), which permits unrestricted use, distribution, and reproduction in any medium, provided you give appropriate credit to the original author(s) and the source, provide a link to the Creative Commons license, and indicate if changes were made.

\section{References}

1. Sariali E, Leonard P, Mamoudy P (2008) Dislocation after total hip arthroplasty using Hueter anterior approach. J Arthroplasty 23:266-272

2. York P, Smarck C, Judet T, Mauffrey C (2016) Total hip arthroplasty via the anterior approach: tips and tricks for primary and revision surgery. Int Orthop. doi:10.1007/s00264-016-3125-3

3. Kobayashi H, Homma Y, Baba T, Ochi H, Matsumoto M, Yuasa T, Kaneko K (2015) Surgeons changing the approach for total hip arthroplasty from posterior to direct anterior with fluoroscopy should consider potential excessive cup anteversion and flexion implantation of the stem in their early experience. Int Orthop. doi:10.1007/s00264-015-3059-1

4. Lee G, Marconi D (2015) Complications following direct anterior hip procedures: costs to both patients and surgeons. J Arthroplasty. doi:10.1016/j.arth.2015.03.043 
5. Jewett BA, Collis DK (2011) High complication rate with anterior total hip arthroplasties on a fracture table. Clin Orthop Relat Res 469:503-507

6. Spaans AJ, van den Hout JAAM, Bolder SBT (2012) High complication rate in the early experience of minimally invasive total hip arthroplasty by the direct anterior approach. Acta Orthop 83:342-346

7. Homma Y, Baba T, Sano K, Ochi H, Matsumoto M, Kobayashi H, Yuasa T, Maruyam Y, Kaneko K (2015) Lateral femoral cutaneous nerve injury with the direct anterior approach for total hip arthroplasty. Int Orthop. doi:10.1007/s00264-015-2942-0

8. Gao Y, Li S, Wang Y, Yang C, Liu J, Qi X (2015) Arthroplasty in patients with extensive femoral head avascular necrosis: cementless arthroplasty in extensive femoral head necrosis. Int Orthop 39: $1507-1511$

9. Schmidutz F, Steinbrück A, Wanke-Jellinek L, Pietschmann M, Jansson V, Fottner A (2012) The accuracy of digital templating: a comparison of short-stem total hip arthroplasty and conventional total hip arthroplasty. Int Orthop 36(9):1767-1772

10. Kniesel B, Konstantinidis L, Hirschmüller A, Südkamp N, Helwig P (2014) Digital templating in total knee and hip replacement: an analysis of planning accuracy. Int Orthop 38:733-739

11. Sariali E, Mauprivez R, Khiami F, Pascal Moussellard H, Catonne Y (2012) Accuracy of the preoperative planning for cementless total hip arthroplasty. A randomised comparison between threedimensional computerised planning and conventional templating. Orthop Traumatol Surg Res 98:151-158

12. Hassani H, Cherix S, Ek E, Rüdiger H (2014) Comparisons of preoperative three-dimensional planning and surgical reconstruction in primary cementless total hip arthroplasty. J Arthroplasty. doi:10.1016/j.arth.2013.12.033

13. Sariali E, Mouttet A, Pasquier G, Durante E, Catonne Y (2009) Accuracy of reconstruction of the hip using computerised three- dimensional pre-operative planning and a cementless modularneck stem. J Bone Joint Surg (Br) 91:333-340

14. Sariali E, Boukhelifa N, Catonne Y, Pascal Moussellard H (2016) Comparison of three-dimensional planning assisted and conventional acetabular cup positioning in total hip arthroplasty: a randomized controlled trial. JBJS Am 98:108-116

15. Huppertz A, Lembcke A, Sariali E, Durmus T, Schwenke C, Hamm B, Sparmann M, Baur A (2015) Low dose computed tomography for 3D planning of total hip arthroplasty: evaluation of radiation exposure and image quality. J Comput Assist Tomogr 39:649-656

16. Sariali E, Klouche S, Mamoudy P (2012) Investigation into three dimensional hip anatomy in anterior dislocation after THA. Influence of the position of the hip rotation centre. Clin Biomech 27:562-567

17. Ji W, Stewart N (2016) Fluoroscopy assessment during anterior minimally invasive hip replacement is more accurate than with the posterior approach. Int Orthop 40:21-27

18. D'Arrigo C, Speranza A, Monaco E, Carcangiu A, Ferretti A (2009) Learning curve in tissue sparing total hip replacement: comparison between different approaches. J Orthop Traumatol 10:4754

19. Flecher X, Parrate S, Ryembault E, Aubaniac J (2006) Custom cementless stem for osteoarthritis following developmental hip dysplasia. Rev Chir Orthop Reparatrice Appar Mot 332-342

20. Sariali E, Klouche S, Mamoudy P (2012) Ceramic-on-ceramic total hip arthroplasty: is squeaking related to an inaccurate threedimensional hip anatomy reconstruction? Orthop Traumatol Surg Res 100:437-440

21. Kop A, Swarts E (2009) Corrosion of a hip stem with a modular neck taper junction: a retrieval study of 16 cases. J Arthroplasty 24: 1019-1023 